\title{
Notificación de Accidentes de Trabajo y Posibles Enfermedades Profesionales en Ecuador, 2010-2015
}

\section{NOTIFICATION OF OCCUPATIONAL ACCIDENTS AND POSSIBLE OCCUPATIONAL DISEASES IN ECUADOR, 2010-2015}

\author{
Antonio Ramón Gómez Garcia', Alfonso Francisco Algora Buenafé2, Pablo Roberto Suasnavas Bermúdez', \\ Michael Giovanny Silva Peñaherrera', Aimee Vilaret Serpa' \\ 1. Facultad de Ciencias del Trabajo y Comportamiento Humano. Universidad Internacional SEK. Quito, Ecuador. \\ 2. Facultad de Derecho. Universidad Internacional SEK. Quito, Ecuador.
}

\section{RESUMEN}

Objetivo: Describir la evolución de notificaciones por accidentes de trabajo y posibles enfermedades profesionales en Ecuador para el período desde 2010 a 2015.

Materiales y Métodos: Estudio descriptivo a partir de fuentes secundarias reportadas al Seguro General de Riesgos de Trabajo del Instituto Ecuatoriano de Seguridad Social; se emplean frecuencias absolutas (n) y relativas (\%), así como el cálculo de la tasa de incidencia en relación con las variables de interés.

Resultados: Se evidencia un incremento considerable del número de casos notificados por accidentes de trabajo y posibles enfermedades profesionales; la tasa de incidencia por accidentes aumentó de 381,2 en 2010 a 775,0 en 2015; asimismo, para los casos de enfermedades de 6,0 en 2010 a 28,4 en 2015.

Conclusiones: El aumento de registros de accidentes de trabajo y posibles enfermedades profesionales al Seguro General de Riesgos de Trabajo podría deberse a la entrada en vigor de normativas sobre la obligatoriedad de notificación en 2010 y 2011. No obstante, se evidencia la falta de implantación de medidas preventivas en los centros de trabajo, lo que supone plantearse una nueva reformulación de las políticas nacionales en seguridad y salud en el trabajo.

(Gómez A, Algora A, Suasnavas P, Silva M, Vilaret A, 2016. Notificación de Accidentes de Trabajo y Posibles Enfermedades Profesionales en Ecuador, 2010-2015. Cienc Trab. Sep-Dic; 18 [57]: 166-172).

Palabras clave: NOTIFICACIÓN DE ACCIDENTES DE TRABAJO; ENFERMEDADES PROFESIONALES; SALUD LABORAL.

\section{ABSTRACT}

Objective: To describe the evolution of reports of occupational accidents and possible occupational diseases in Ecuador for the period from 2010 to 2015.

Materials and Methods: Descriptive study from secondary sources reported to the General Occupational Risk Insurance of the Ecuadorian Social Security Institute; Absolute (n) and relative frequencies (\%) are used, as well as the calculation of the incidence rate in relation to the variables of interest.

Results: There is a considerable increase in the number of cases reported due to occupational accidents and possible occupational diseases; The incidence rate for accidents increased from 381.2 in 2010 to 775.0 in 2015; Likewise, for cases of diseases from 6.0 in 2010 to 28.4 in 2015.

Conclusions: The increase of records of occupational accidents and possible occupational diseases to the General Insurance of Occupational Risks could be due to the regulations on the obligation of notification that became effective in 2010 and 2011. However, it is evident the lack of implantation of preventive measures in the work centers, what supposes to consider a new reformulation of the national policies in security and health in the work.

Keywords: NOTIFICATION OF WORK ACCIDENTS; OCCUPATIONAL DISEASES; OCCUPATIONAL HEALTH.

\section{INTRODUCCIÓN}

La Organización Internacional del Trabajo (OIT) estima que, a nivel mundial, cada 15 segundos se producen 153 accidentes de trabajo, causándole la muerte a 1 trabajador, derivándose anualmente en 2,3 millones de fallecimientos, 270 millones de acci-

Correspondencia / Correspondence:

Ph. D Antonio Ramón Gómez García

Universidad Internacional SEK

Campus Miguel de Cervantes. Alberto Einstein, s/n y 5 ta transversal.

Quito, Ecuador.

Tel.: +593 3974800 Ext. 169

e-mail: antonio.gomez@uisek.edu.ec

Recibido: 02 de Agosto / Aceptado 29 de Septiembre de 2016 dentes de trabajo y 160 millones de enfermedades profesionales, siendo el costo económico estimado del $4 \%$ del producto interno bruto mundial ${ }^{1}$, lo que genera un impacto en las economías de los países $^{2,4}$, en la productividad de las empresas ${ }^{5}$ y un problema en salud pública. ${ }^{6-7}$

En los últimos años, la Organización Iberoamericana de Seguridad Social (OISS) ha realizado grandes esfuerzos en armonizar los datos de siniestralidad laboral para los países miembros. En 2006, estableció una línea de trabajo dirigida a impulsar las políticas nacionales en materia de seguridad y salud en el trabajo, creando el Observatorio de Siniestralidad Laboral ${ }^{8}$ con el objetivo de mejorar los sistemas de información, registro e indicadores estadísticos de accidentes de trabajo y enfermedades profesionales para los países iberoamericanos ${ }^{9,10}$; no obstante, los datos consultados en el Sistema Armonizado de Indicadores de Siniestralidad Laboral en Iberoamérica (SIARIN) ${ }^{11}$ no están actualizados o completos para la mayoría de los países. 
En Ecuador, si bien existen investigaciones publicadas ${ }^{12,13}$ en la última década que ofrecen información sobre el diagnóstico situacional sobre la seguridad y salud en el trabajo, son escasos los documentos encontrados que permitan evaluar la tendencia y evolución de la siniestralidad laboral más reciente en este país $^{14,15}$; asimismo, los datos disponibles por el Seguro General de Riesgos de Trabajo (SGRT) ${ }^{16}$ del Instituto Ecuatoriano de Seguridad Social (IESS) abarcan un período relativamente corto, de 2013 hasta 2016 (avance mensual).

Los accidentes de trabajo y enfermedades profesionales están reconocidos legalmente ${ }^{16-17}$ en el Ecuador, así como las prestaciones y subsidios por incapacidad laboral, normativas que tiene su origen en convenios y resoluciones internacionales ${ }^{18-20}$ adoptadas por este país. El empleador está obligado a notificar ${ }^{21}$ al SGRT los Accidentes de Trabajo (A.T.) y posibles Enfermedades Profesionales (E.P.) ocurridos en los centros de trabajo, para la correspondiente calificación a través del Comité de Valuación de Incapacidades y de Responsabilidad Patronal (CVIRP), así como para la verificación del grado de implantación de las medidas preventivas en los centros de trabajo ${ }^{22}$ y la correspondiente responsabilidad patronal del empleador ${ }^{23}$.

En este contexto de estudio, el A.T. ${ }^{21}$ es considerado como aquel suceso imprevisto y repentino que sobrevenga por causa, consecuencia o con ocasión del trabajo originado por la actividad laboral relacionada con el puesto de trabajo, que ocasione en el afiliado (trabajador) lesión corporal o perturbación funcional, una incapacidad, o la muerte inmediata o posterior. La E.P. ${ }^{21}$ es aquella afección crónica, causada de una manera directa por el ejercicio de la profesión u ocupación que realiza el trabajador y como resultado de la exposición a factores de riesgo, que producen o no incapacidad laboral.

Considerando los antecedentes anteriormente expuestos y la necesidad de disponer de información actualizada sobre la siniestralidad laboral en Ecuador que permita un posterior análisis y evaluación de las políticas públicas en este ámbito y la comparación con años anteriores ${ }^{24}$, el objetivo del presente estudio fue describir la evolución de las notificaciones de accidentes de trabajo y enfermedades profesionales notificadas al SGRT en el período desde 2010 a 2015 .

\section{MATERIAL Y MÉTODOS}

Estudio de tipo descriptivo, se describen las frecuencias absolutas (n) y relativas (\%n) de las notificaciones de accidentes de trabajo y posibles enfermedades profesionales reportadas por el empleador al Seguro General de Riesgos de Trabajo ${ }^{15}$ del Instituto Ecuatoriano de Seguridad Social.

Asimismo, se calcula la Tasa de Incidencia (T.I.) para cada variable analizada mediante la fórmula establecida por Eurostat ${ }^{26}$, metodología empleada por los países pertenecientes a la Unión Europea y que permite la comparación internacional ${ }^{7-9 ; 26}$, siendo el numerador el número de casos notificados por accidente de trabajo y posible enfermedad profesional x $100.000 \mathrm{y}$, el denominador corresponde al número de trabajadores afiliados al Seguro General Obligatorio ${ }^{27-28}$ del IESS para cada período de estudio, que incluye a trabajadores en relación de dependencia, autónomos y profesionales en libre ejercicio.

Una primera fase consistió en la recopilación de la información ${ }^{15-29}$, confección de una base de datos en SPSS Statistics (versión 23) y depuración de calidad de los mismos. En función de esta información, las variables se clasificaron en provincias (24), accidentes de trabajo y posibles enfermedades profesionales notificadas. En una segunda fase se procedió al tratamiento estadístico en función del objeto de estudio; asimismo, se usó el generador de gráficos Microsoft Excel 2011 para representar la tendencia lineal de las notificaciones de A.T. y E.P.

Este estudio no requirió la aprobación de un comité de ética por tratarse de un análisis de datos a partir de información secundaria y de libre dominio público ${ }^{29,30}$ en la que no se identifican a los sujetos cuyos casos fueron reportados por el empleador como accidentes de trabajo y/o posibles enfermedades profesionales.

\section{RESULTADOS}

La población afiliada al Instituto Ecuatoriano de Seguridad Social (IESS) desde 2010 a 2015 se incrementó en 1.072.298 trabajadores afiliados, registrándose un máximo de 3.386.721 en 2013. Las provincias con mayor concentración de trabajadores en 2015 son: Pichincha, con 1.069.032 (34,0\%) afiliados, Guayas con 843.824 (26,8\%), Manabí con 223.309 (7,1\%), Azuay con 184.401 (5,9\%), El Oro con 110.039 (3,5\%) y 93.240 (3,0\%) afiliados la provincia de Tungurahua, Tabla 1.

En 2015, se observa un descenso de afiliados (-241.117) respecto a 2014, siendo las provincias de Santa Elena (-56.242), Los Ríos $(-34.680)$ y Pichincha (-30.698) las más destacadas.

Para el periodo de estudio, se notificaron un total de 99.156 A.T. y 2.733 casos de posibles E.P. al Seguro General de Riesgos de Trabajo del IESS, registrándose en 2015 el mayor número de notificaciones en ambos casos (24.379 y 892). En 2013, respecto a 2012, se observa un aumento considerable en las notificaciones de A.T. (7.128). La Tabla 2 muestra la distribución de notificaciones por accidentes de trabajo desde 2010 a 2015. Las provincias con mayor número de notificaciones por A.T. registrados en este período corresponden a Guayas con 44.343 (44,7\%), Pichincha con 22.766 (23,0\%), Los Ríos 4.333 (4,4\%) y con 3.987 $(4,0 \%)$ casos notificados la provincia de Azuay. Los 23.727 (24\%) casos restantes por A.T. se distribuyen en el resto de provincias del país. Destacan Galápagos con $146(0,1 \%)$ y Carchi con 241 $(1,0 \%)$ por su baja siniestralidad laboral.

En el año 2010 se notificó un total de 7.904 accidentes de trabajo (A.T.). Las provincias con mayor número de notificaciones corresponden a Guayas con 3.371 (42,6\%) y Pichincha con 1.471 $(18,6 \%)$. Respecto a 2011, se notificaron 9.338 casos, manteniéndose Guayas con 4.181 (44,8\%) y Pichincha con 2.005 (21,5\%) como las provincias de mayor porcentaje de casos a nivel nacional. En 2012, el total de notificaciones fue de 13.657. Para las provincias de Los Ríos, Pastaza y Zamora Chinchipe no se observan registros de A.T. en las Direcciones Provinciales del SGRT. Guayas con $6.801(49,8 \%)$ y Pichincha con $3.352(24,5 \%)$ se mantienen en orden de importancia. Respecto a 2013, se notificaron 20.785 casos de A.T.: Guayas con 9.758 (46,9\%), Pichincha con 4.671 (22,5\%), Los Ríos con $952(4,6 \%)$ y con $709(3,4 \%)$ Azuay, como las principales provincias.

En 2014 se notificaron al SGRT un total de 23.093 de A.T. Destacan en orden de importancia Guayas con 9.911 (42,9\%), Pichincha con 5.560 (24,1\%), Los Ríos con 1.022 (4,4\%), Manabí con 963 (4,2\%) y con $797(3,5 \%)$ casos de notificaciones de A.T. la provincia de Azuay. Las provincias con menor número de casos notificados en 
Tabla 1.

Distribución de la población afiliada al IESS por año y provincia, 2010-2015.

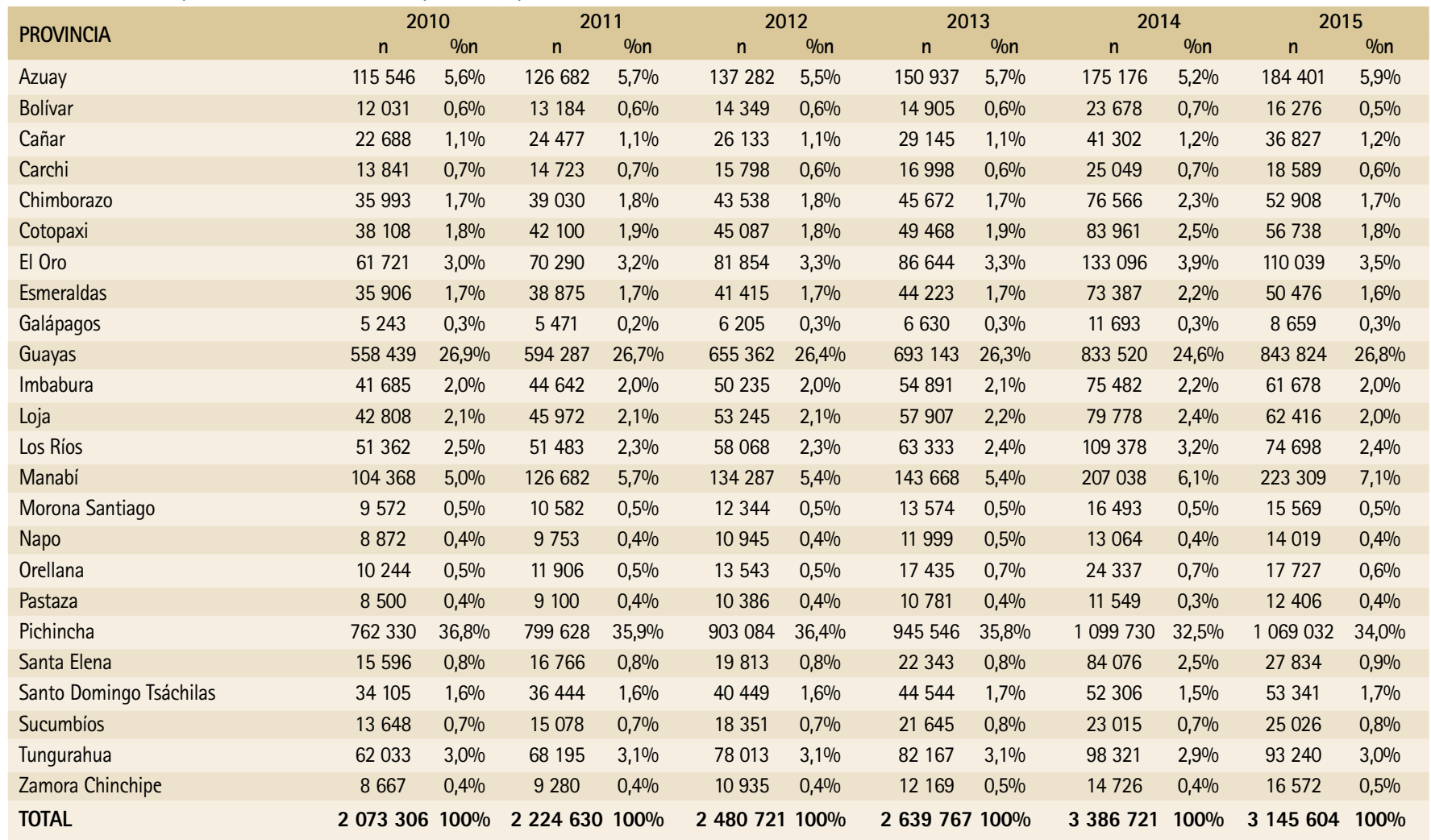

Tabla 2.

Distribución de notificaciones por A.T. por año y provincia, 2010-2015.

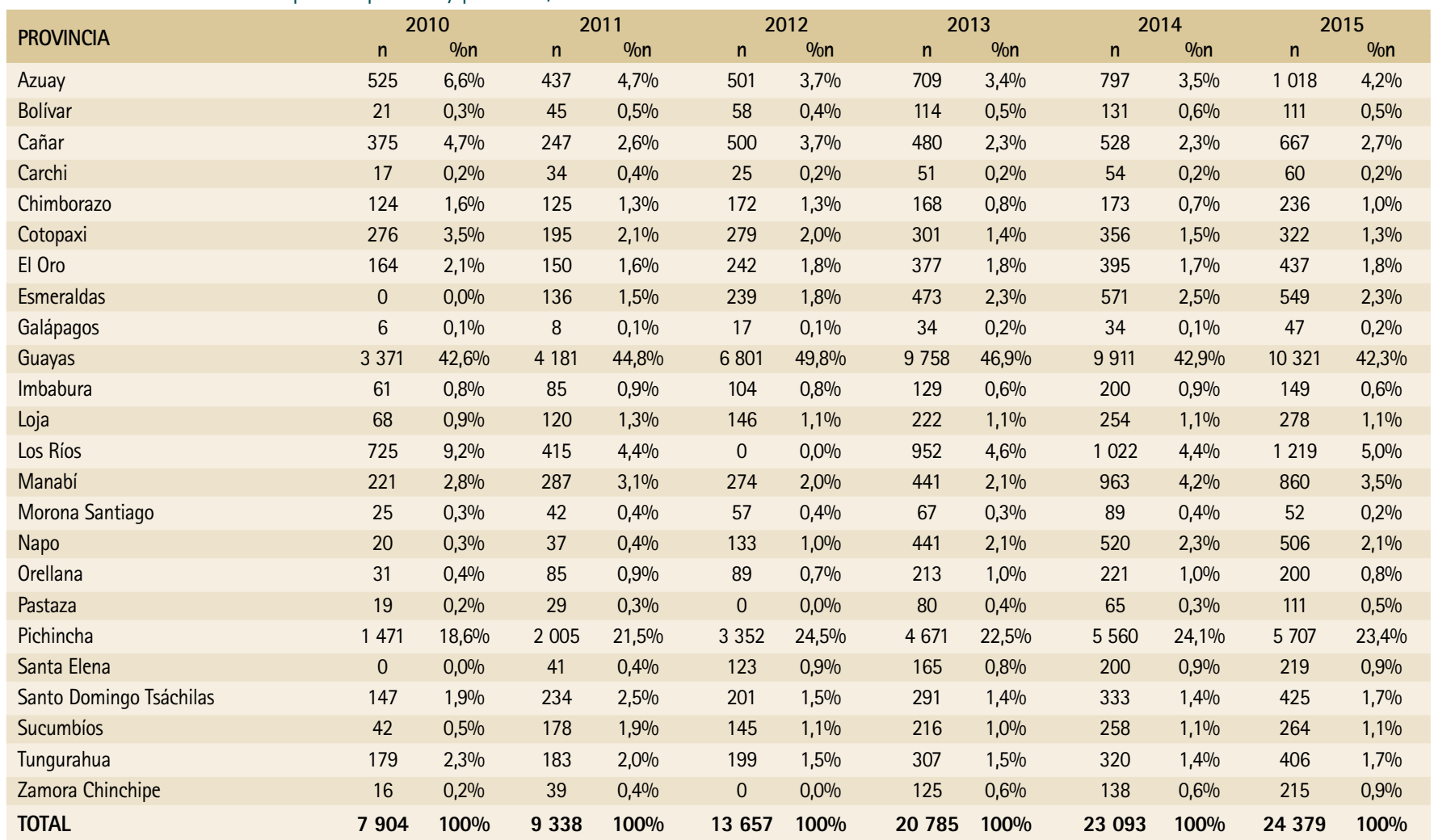


este año son Galápagos con $34(0,1 \%)$, Pastaza con 65 (0,3\%) y con $89(0,4 \%)$ A.T. la provincia de Morona Santiago.

Para el último año de estudio analizado, se notificaron un total del 24.379 A.T.: Guayas con 10.321 (42,3\%), Pichincha con 5.707 (23,4\%), Los Ríos con 1.219 (5,0\%) y Azuay con 1.018 (4,2\%) como las provincias con mayor notificación, siendo Galápagos con 47, Morona Santiago con 52 y 60 la provincia de Carchi las provincias con menor número de casos notificados $(0,2 \%)$.

En cuanto a las notificaciones de posibles enfermedades profesionales (E.P.), se observa un incremento importante: 124 notificaciones en 2010 respecto a 892 casos en 2015. En la Tabla 3, se presenta la distribución de notificaciones de posibles enfermedades profesionales por año y provincia.

Para el año 2010 no se dispone de información de E.P. por provincias; no obstante, a nivel nacional se notificaron un total de 124 casos. En 2011, el total de E.P. fue de 177 notificados, solamente las provincias de Pichincha con 156 (88,1\%), Guayas con 12 (6,8\%) y $9(5,1 \%)$ Azuay reportaron casos en las respectivas Direcciones Provinciales del SGRT.

Respecto a 2012, se notificaron 206 casos de posibles E.P. Las provincias con mayor registro fueron Pichincha con 121 (58,7\%), Cotopaxi con 22 (10,7\%), Guayas con $14(6,8 \%)$ y $14(6,8 \%)$ casos la provincia de Imbabura. Para 2013, el total de casos fue de 630, Pichincha con 356 (56,5\%) de E.P., Guayas con 72 (11,4\%), Cotopaxi con $48(7,6 \%)$ y $32(5,1 \%)$ casos la provincia de Orellana. Asimismo, 740 casos se notificaron en 2014, registrándose 394 (56,0\%) en Pichincha y $83(11,8 \%)$ en Guayas como las provincias de mayor número de casos notificados de E.P.

Por último, en 2015 se notificaron un total de 892 casos de posibles E.P., 715 casos más que los registrados en 2010. Las provincias de Pichincha (535) y Guayas (98) conformaron el 71\% del total de notificaciones.

Cabe mencionar que las provincias de Bolivar (7), Carchi (3) y Morona Santiago (1) son las de menor número de casos notificados de posibles E.P. para el período de 2011 a 2015 a nivel nacional.

$\mathrm{Al}$ calcular las tasas de incidencia por notificaciones de accidentes de trabajo y posibles enfermedades profesionales, se observó un incremento importante para el período de estudio en la T.I.A.T. (381,2 el 2010; 775,0 el 2015) y la T.I.E.P. (6,0 el 2010; 28,4 el 2015). El año de mayor incidencia de notificaciones por accidentes de trabajo con 787,4 por cada 100.000 trabajadores afiliados fue 2013 , seguido de 2015 con 775,0. Asimismo, la mayor incidencia de casos por posible enfermedad profesional fue de 28,4 en 2015. En la Tabla 4, se presentan las tasas de incidencia para accidentes de trabajo y posibles enfermedades profesionales por año y provincia.

En 2010, la T.I.A.T más alta se registró en las provincias de Cañar (1.652,9 casos por cada 100.000 afiliados) y Los Ríos (1.411,5 casos por cada 100.000 afiliados). Para 2011, Sucumbíos (1180,5) y Cañar $(1009,1)$ registraron la incidencia más importante respecto al resto de provincias.

Respecto al 2012, las provincias de Napo (1215,2 casos por cada 100.000 afiliados) y Cañar (1913,3 casos por cada 100.000 afiliados) registraron la T.I.A.T más alta; asimismo, en 2013 destacan Napo (3675,3), Cañar (1646,9), Los Ríos (1503,2), Guayas $(1407,8)$, Orellana $(1221,7)$, Esmeraldas $(1069,6)$ y Zamora Chinchipe $(1027,2)$.

Las Figuras 1 y 2 muestran la tendencia de las notificaciones de accidentes de trabajo y enfermedades profesionales desde 2010 a 2015. Se observa una tendencia al aumento de reportes al Seguro General de Riesgos del Trabajo.

Tabla 3.

Distribución de notificaciones de posibles E.P. por año y provincia, 2011-2015.

\begin{tabular}{|c|c|c|c|c|c|c|c|c|c|c|}
\hline \multirow{2}{*}{ PROVINCIA } & \multicolumn{2}{|c|}{2011} & \multicolumn{2}{|c|}{2012} & \multicolumn{2}{|c|}{2013} & \multicolumn{2}{|c|}{2014} & \multicolumn{2}{|c|}{2015} \\
\hline & $n$ & $\% n$ & $n$ & $\% n$ & $\mathrm{n}$ & \%n & $\mathrm{n}$ & \%n & $n$ & $\%$ n \\
\hline Azuay & 9 & $5,1 \%$ & 1 & $0,5 \%$ & 11 & $1,7 \%$ & 19 & $2,7 \%$ & 13 & $1,5 \%$ \\
\hline Bolivar & 0 & $0,0 \%$ & 1 & $0,5 \%$ & 0 & $0,0 \%$ & 3 & $0,4 \%$ & 3 & $0,3 \%$ \\
\hline Cañar & 0 & $0,0 \%$ & 0 & $0,0 \%$ & 2 & $0,3 \%$ & 4 & $0,6 \%$ & 5 & $0,6 \%$ \\
\hline Carchi & 0 & $0,0 \%$ & 0 & $0,0 \%$ & 1 & $0,2 \%$ & 0 & $0,0 \%$ & 2 & $0,2 \%$ \\
\hline Chimborazo & 0 & $0,0 \%$ & 4 & $1,9 \%$ & 0 & $0,0 \%$ & 2 & $0,3 \%$ & 19 & $2,1 \%$ \\
\hline Cotopaxi & 0 & $0,0 \%$ & 22 & $10,7 \%$ & 48 & $7,6 \%$ & 38 & $5,4 \%$ & 29 & $3,3 \%$ \\
\hline El Oro & 0 & $0,0 \%$ & 2 & $1,0 \%$ & 5 & $0,8 \%$ & 2 & $0,3 \%$ & 11 & $1,2 \%$ \\
\hline Esmeraldas & 0 & $0,0 \%$ & 2 & $1,0 \%$ & 3 & $0,5 \%$ & 3 & $0,4 \%$ & 4 & $0,4 \%$ \\
\hline Galápagos & 0 & $0,0 \%$ & 0 & $0,0 \%$ & 7 & $1,1 \%$ & 7 & $1,0 \%$ & 0 & $0,0 \%$ \\
\hline Guayas & 12 & $6,8 \%$ & 14 & $6,8 \%$ & 72 & $11,4 \%$ & 83 & $11,8 \%$ & 98 & $11,0 \%$ \\
\hline Imbabura & 0 & $0,0 \%$ & 14 & $6,8 \%$ & 13 & $2,1 \%$ & 17 & $2,4 \%$ & 12 & $1,3 \%$ \\
\hline Loja & 0 & $0,0 \%$ & 9 & $4,4 \%$ & 23 & $3,7 \%$ & 26 & $3,7 \%$ & 16 & $1,8 \%$ \\
\hline Los Ríos & 0 & $0,0 \%$ & 1 & $0,5 \%$ & 3 & $0,5 \%$ & 5 & $0,7 \%$ & 5 & $0,6 \%$ \\
\hline Manabi & 0 & $0,0 \%$ & 0 & $0,0 \%$ & 2 & $0,3 \%$ & 13 & $1,8 \%$ & 9 & $1,0 \%$ \\
\hline Morona Santiago & 0 & $0,0 \%$ & 0 & $0,0 \%$ & 0 & $0,0 \%$ & 0 & $0,0 \%$ & 1 & $0,1 \%$ \\
\hline Napo & 0 & $0,0 \%$ & 2 & $1,0 \%$ & 19 & $3,0 \%$ & 6 & $0,9 \%$ & 13 & $1,5 \%$ \\
\hline Orellana & 0 & $0,0 \%$ & 3 & $1,5 \%$ & 32 & $5,1 \%$ & 9 & $1,3 \%$ & 34 & $3,8 \%$ \\
\hline Pastaza & 0 & $0,0 \%$ & 0 & $0,0 \%$ & 0 & $0,0 \%$ & 4 & $0,6 \%$ & 10 & $1,1 \%$ \\
\hline Pichincha & 156 & $88,1 \%$ & 121 & $58,7 \%$ & 356 & $56,5 \%$ & 394 & $56,0 \%$ & 535 & $60,0 \%$ \\
\hline Santa Elena & 0 & $0,0 \%$ & 0 & $0,0 \%$ & 2 & $0,3 \%$ & 5 & $0,7 \%$ & 5 & $0,6 \%$ \\
\hline Santo Domingo Tsáchilas & 0 & $0,0 \%$ & 6 & $2,9 \%$ & 2 & $0,3 \%$ & 19 & $2,7 \%$ & 12 & $1,3 \%$ \\
\hline Sucumbios & 0 & $0,0 \%$ & 2 & $1,0 \%$ & 10 & $1,6 \%$ & 21 & $3,0 \%$ & 27 & $3,0 \%$ \\
\hline Tungurahua & 0 & $0,0 \%$ & 2 & $1,0 \%$ & 14 & $2,2 \%$ & 16 & $2,3 \%$ & 21 & $2,4 \%$ \\
\hline Zamora Chinchipe & 0 & $0,0 \%$ & 0 & $0,0 \%$ & 5 & $0,8 \%$ & 8 & $1,1 \%$ & 8 & $0,9 \%$ \\
\hline TOTAL & 177 & $100 \%$ & 206 & $100 \%$ & 630 & $100 \%$ & 704 & $100 \%$ & 892 & $100 \%$ \\
\hline
\end{tabular}


Figura 1.

Tendencia de notificaciones de accidentes de trabajo, 2010-2015.

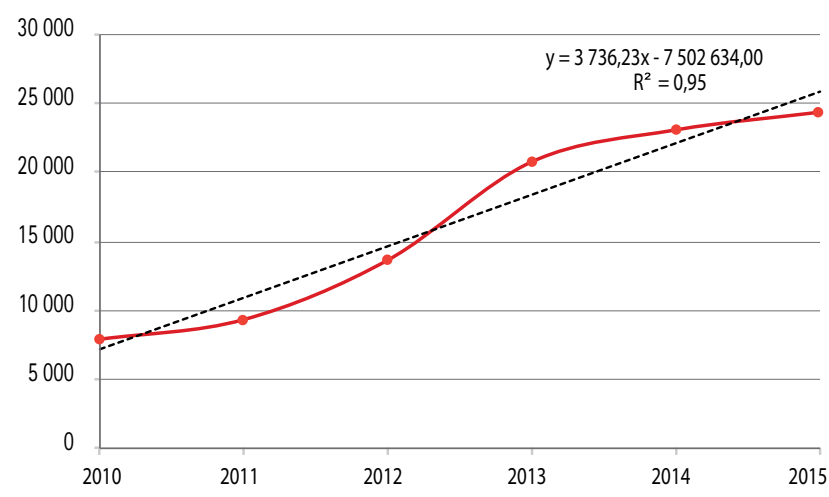

En 2014, las provincias de mayor incidencia se registraron en las provincias de Napo $(3980,4)$, Cañar $(1278,4)$, Guayas $(1189,1)$ y Sucumbíos (1121,0). Por último, para el año 2015 destaca la provincia de Napo con 3609,4 casos por cada 100.000 afiliados, seguido de Cañar $(1811,2)$, Los Ríos $(1631,9)$, Zamora Chinchipe $(1297,4)$ y Guayas $(1223,1)$.

En cuanto a la tasa de incidencia por notificaciones de posibles enfermedades profesionales, en 2010 no se disponen de datos sobre trabajadores afiliados y E.P. que permitieran realizar el cálculo de la T.I.E.P. En 2011, solo tres provincias notificaron posibles casos de enfermedades profesionales: Pichincha (19,5 casos por cada
Figura 2.

Tendencia de notificaciones de posibles enfermedades profesionales, 2010-2015.

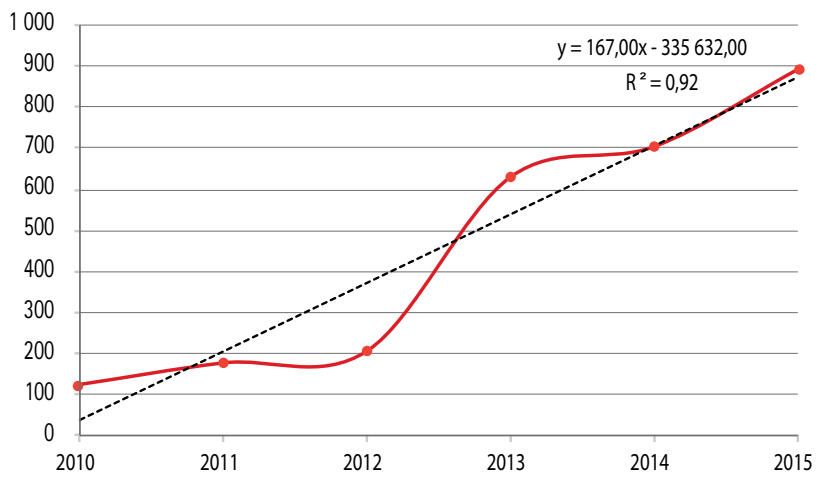

100.000 afiliados), Azuay (7,1 casos por cada 100.000 afiliados) y Guayas (2,0 casos por cada 100.000 afiliados).

Respecto a 2012, destacan las provincias de Cotopaxi con 48,8 casos por cada 100.000 afiliados, seguida de Imbabura con 27,9. En 2013, las mayores incidencias se registraron en las provincias de Orellana $(183,5)$, Napo $(158,3)$ y Galápagos $(105,6)$. En 2014, Sucumbíos $(91,2)$, Galápagos $(59,9)$ y Zamora Chinchipe $(54,3)$ conforman la mayor tasa de incidencia por notificaciones de posibles enfermedades profesionales a nivel nacional; por último, en 2015 fueron, en orden de tasa de incidencia, las provincias de Orellana $(191,8)$, Sucumbíos $(107,9)$ y Napo $(92,7)$.

Tabla 4.

Distribución de T.I.A.T. y TI.E.P. por 100.000 trabajadores afiliados, por año y provincia, 2010-2015.

\begin{tabular}{|c|c|c|c|c|c|c|c|c|c|c|c|c|}
\hline PROVINCIA & \multicolumn{2}{|c|}{2010} & \multicolumn{2}{|c|}{2011} & \multicolumn{2}{|c|}{2012} & \multicolumn{2}{|c|}{2013} & \multicolumn{2}{|c|}{2014} & \multicolumn{2}{|c|}{2015} \\
\hline Bolivar & 174,5 & * & 341,3 & 0,0 & 404,2 & 7,0 & 764,8 & 0,0 & 553,3 & 12,7 & 682,0 & 18,4 \\
\hline Carchi & 122,8 & * & 230,9 & 0,0 & 158,2 & 0,0 & 300,0 & 5,9 & 215,6 & 0,0 & 322,8 & 10,8 \\
\hline Chimborazo & 344,5 & * & 320,3 & 0,0 & 395,1 & 9,2 & 367,8 & 0,0 & 225,9 & 2,6 & 446,1 & 35,9 \\
\hline Cotopaxi & 724,3 & * & 463,2 & 0,0 & 618,8 & 48,8 & 608,5 & 97,0 & 424,0 & 45,3 & 567,5 & 51,1 \\
\hline Esmeraldas & 0,0 & * & 349,8 & 0,0 & 577,1 & 4,8 & 1069,6 & 6,8 & 778,1 & 4,1 & 1087,6 & 7,9 \\
\hline Galápagos & 114,4 & * & 146,2 & 0,0 & 274,0 & 0,0 & 512,8 & 105,6 & 290,8 & 59,9 & 542,8 & 0,0 \\
\hline Guayas & 603,6 & * & 703,5 & 2,0 & 1037,7 & 2,1 & 1407,8 & 10,4 & 1189,1 & 10,0 & 1223,1 & 11,6 \\
\hline Imbabura & 146,3 & * & 190,4 & 0,0 & 207,0 & 27,9 & 235,0 & 23,7 & 265,0 & 22,5 & 241,6 & 19,5 \\
\hline Loja & 158,8 & * & 261,0 & 0,0 & 274,2 & 16,9 & 383,4 & 39,7 & 318,4 & 32,6 & 445,4 & 25,6 \\
\hline Los Ríos & 1411,5 & * & 806,1 & 0,0 & 0,0 & 1,7 & 1503,2 & 4,7 & 934,4 & 4,6 & 1631,9 & 6,7 \\
\hline Orellana & 302,6 & * & 713,9 & 0,0 & 657,2 & 22,2 & 1221,7 & 183,5 & 908,1 & 37,0 & 1128,2 & 191,8 \\
\hline Pastaza & 223,5 & * & 318,7 & 0,0 & 0,0 & 0,0 & 742,0 & 0,0 & 562,8 & 34,6 & 894,7 & 80,6 \\
\hline Pichincha & 193,0 & * & 250,7 & 19,5 & 371,2 & 13,4 & 494,0 & 37,7 & 505,6 & 35,8 & 533,8 & 50,0 \\
\hline Santa Elena & 0,0 & $*$ & 244,5 & 0,0 & 620,8 & 0,0 & 738,5 & 9,0 & 237,9 & 5,9 & 786,8 & 18,0 \\
\hline Santo Domingo Tsáchilas & 431,0 & * & 642,1 & 0,0 & 496,9 & 14,8 & 653,3 & 4,5 & 636,6 & 36,3 & 796,8 & 22,5 \\
\hline Sucumbios & 307,7 & * & 1180,5 & 0,0 & 790,1 & 10,9 & 997,9 & 46,2 & 1121,0 & 91,2 & 1054,9 & 107,9 \\
\hline Tungurahua & 288,6 & * & 268,3 & 0,0 & 255,1 & 2,6 & 373,6 & 17,0 & 325,5 & 16,3 & 435,4 & 22,5 \\
\hline Zamora Chinchipe & 184,6 & * & 420,3 & 0,0 & 0,0 & 0,0 & 1027,2 & 41,1 & 937,1 & 54,3 & 1297,4 & 48,3 \\
\hline
\end{tabular}

T.I.A.T. Tasa de incidencia por accidentes de trabajo. T.I.E.P. Tasa de incidencia por posibles enfermedades profesionales. ${ }^{*}$ Sin datos. 


\section{DISCUSIÓN}

El presente estudio sobre la evolución de las notificaciones de accidentes de trabajo y posibles enfermedades profesionales, reportadas al Seguro General de Riesgos del Trabajo del Instituto Ecuatoriano de Seguridad Social para el período desde 2010 a 2015, ofrece información sobre la realidad actual de la siniestralidad laboral en el país 9 .

Se observa, en los últimos tres años, un incremento considerable del número de casos notificados por accidentes de trabajo (7.904 en 2010 a 24.379 en 2015) y posibles enfermedades profesionales (177 en 2010 a 892 en 2015), lo que podría deberse a la entrada en vigor del Reglamento para el Sistema de Auditoría de Riesgos del Trabajo ${ }^{31}$ en 2010 y el Reglamento del Seguro General de Riesgos del Trabajo ${ }^{32}$ en 2011, aunque actualmente derogadas ${ }^{21}$, obligaban al empleador a notificar, en el término de diez (10) días, la ocurrencia de accidentes de trabajo y/o presunción de enfermedad profesional a las Unidades Provinciales del Seguro General de Riesgos del Trabajo. Para el caso de accidentes de trabajo, en el término de treinta (30) días, contados a partir de la fecha del accidente, el empleador estaba obligado a presentar el correspondiente informe sobre la investigación del mismo y las medidas correctivas implantadas.

No obstante, la vigente Resolución No. C.D. 51322 de abril de 2016, además de continuar obligando al empleador a notificar los accidentes y posibles enfermedades, abre un nuevo espacio en seguridad y salud ocupacional para Ecuador. Junto con otras disposiciones legales, esta normativa trata de fortalecer e impulsar la gestión interna de la Dirección General de Riesgos del Trabajo y la creación de una cultura de prevención de riesgos laborales en las empresas mediante los principios de la acción preventiva (Art. 53). Aunque ya se incluían en las Reglamentos derogados, centra sus esfuerzos en potenciar el control de riesgos en su origen, en el medio o finalmente en el receptor; en la planificación preventiva mediante la evaluación de riesgos; en la detección precoz de enfermedades profesionales y la vigilancia periódica de la salud de los trabajadores, entre otros.

A pesar de la normativa vigente, se evidencia que las empresas no aplican los principios de esta acción preventiva: así lo demuestran las gráficas de tendencia. Por cada 100.000 trabajadores afiliados, la tasa de incidencia por accidentes de trabajo aumentó de 381,2 en 2010 a 775,0 en 2015; asimismo, para las posibles enfermedades profesionales de 6,0 a 28,4, respectivamente. Considerando el 2015 como el año de mayor notificación de accidentes de trabajo (24.379), podemos aproximarnos en afirmar que cada día se producen en Ecuador alrededor 67 accidentes de trabajo.

Entre las principales limitaciones de este estudio podemos considerar la subnotificación ${ }^{2,32}$ de accidentes de trabajo y posibles enfermedades profesionales al ente rector de nuestro país, ocasionando que las cifras presentadas no reflejen la realidad nacional. Considerando la estimación de la OIT en la que sólo se declaran entre un 20 y un 25\% de los accidentes de trabajo en países latinoamericanos y en base a los datos analizados en este estudio, se calcula que en 2015 las notificaciones de accidente de trabajo debieron ser 97.516 respecto a $24.379 .3^{33}$

Otra limitación fue la imposibilidad de calcular las tasas según la actividad económica y horas/hombre, debido a que no se contaba con esta información, lo que nos hubiera permitido calcular el impacto según la exposición en los trabajadores.

A pesar de estas limitaciones, consideramos que los resultados presentados en este estudio permiten aproximarnos a la situación sobre la siniestralidad laboral en el país ${ }^{9}$ y, a su vez, facilitar la comparación internacional $^{7-9}$ y evaluar las políticas públicas en la materia.

Si bien los accidentes y enfermedades generan un daño a la salud del trabajador, un impacto económico y productivo en las empresas $^{2-5}$, sería necesario y complementario realizar investigaciones paralelas sobre el costo económico que supone la siniestralidad laboral y estudios sobre los factores y causas que originan los A.T. y E.P. Asimismo, dada la magnitud de este problema, creemos conveniente continuar desarrollando líneas de investigación que permitan identificar con mayor precisión los factores que generan el subregistro, con el objetivo de mejorar la fiabilidad de las estadísticas y, por ende, la calidad de las mismas.

En conclusión, los resultados obtenidos en este estudio evidencian una tendencia al incremento en las notificación de accidentes de trabajo y posibles enfermedades profesionales para los próximos años; por ello, la seguridad y salud en el trabajo en Ecuador se enfrenta a nuevos desafíos y necesidades en la formación de profesionales en este ámbito, así como el desarrollo de un cuerpo legal más estricto para la implantación de medidas preventivas que eliminen o minimicen la probabilidad de sufrir A.T. o E.P. 


\section{REFERENCIAS}

1. Somavia J. Facts on Safety at Work [on line]. Geneva: International Labour Organization; 2005. [consultado 19 feb 2016]. Disponible en: http://www.ilo. org/wcmsp5/groups/public/---dgreports/---dcomm/documents/publication/ wcms_067574.pdf

2. Hämäläinen $P$, Takala J, Saarela KL. Global estimates of fatal work-related diseases. Am J Ind Med. 2007; 50(1):28-41.

3. Leigh JP. Economic burden of occupational injury and illness in the United States Milbank 0 [on line]. 2011; 89(4):728-72 [consultado 6 may 2016]. Disponible en: http://www.ncbi.nlm.nih.gov/pmc/articles/PMC3250639/pdf/ milq0089-0728.pdf

4. Duque-De Voz N, Yánez-Contreras M. Perspectivas diferenciadas del análisis de la accidentalidad laboral. Gaceta Laboral [en línea] 2015; 21(3):313-331 [consultado 6 abr 2016]. Disponible en: http://www.redalyc.org/pdf/737/ 73743964004.pdf

5. Gervais R, Pawlowska Z, Bojanowski R, Murray M, Van den Broek K. Occupational safety and health and economic performance in small and medium-sized enterprises: a review [on line]. Luxembourg: European Agency for Safety and Health at Work; 2009 [cited 14 may 2016]. (Working Environment Information, Working Papers, 9). Available from: https://osha.europa.eu/sites/ default/files/publications/documents/en/publications/reports/TE-80-09-640EN-N_occupational_safety_health_economic_performance_small_medium_ sized_enterprises_review/OSH $\% 20$ SME $\% 20$ REPORT\%20-\%20FINAL $\% 20$ 020609.pdf

6. Concha-Barrientos $M$, Nelson DI, Fingerhut $M$, Driscoll T, Leigh J. The global burden due to occupational injury. Am J Ind Med. 2005; 48(6):470-81.

7. Benavides F, Delclos J, Benach J, Serra C. Lesiones por accidentes de trabajo, una prioridad en salud pública. Rev Esp Salud Pública [en línea]. 2006; 80(5):553565 [consultado 11 jun 2016]. Disponible en: http://scielo.isciii.es/pdf/resp/ v80n5/colaboracion9.pdf

8. Observatorio Iberoamericano de Riesgos Profesionales. SIARIN: Sistema Armonizado de Indicadores de siniestralidad laboral en Iberoamérica [on line]. Madrid: Organización Iberoamericana de Seguridad Social; 2013 [consultado 6 feb 2016]. Disponible en: http://www.oiss.org/estrategia/siarin/

9. Organización Iberoamericana de Seguridad Social. I Estrategia Iberoamericana de Seguridad y Salud en el Trabajo 2010-2013. Madrid: OISS; 2009.

10. Organización Iberoamericana de Seguridad Social. Estrategia Iberoamericana de Seguridad y Salud en el Trabajo 2015-2020 (EISST). Fuentes de Información. SIARIN [en línea] Madrid: OISS; 2014. [consultado 17 feb 2016]. Disponible en: http://www.oiss.org/estrategia/IMG/pdf/Fuentes_de_Informacion.pdf

11. Organización Iberoamericana de Seguridad Social. Estado de situación y fuentes de información. SIARIN [en línea] Madrid: OISS: 2013 [consultado 17 feb 2016]. Disponible en: http://www.oiss.org/estrategia/IMG/pdf/seguimiento_adhesiones.pdf 12. Forastieri V. Perfil diagnóstico en seguridad y salud en el trabajo de los paises de la Subregión Andina: Bolivia, Ecuador, Colombia, Perú y la República Bolivariana de Venezuela. Ginebra: Organización Internacional del Trabajo; 2007. [consultado 17 feb 2016]. Disponible en: http://www.ilo.org/ wcmsp5/groups/public/---ed_protect/---protrav/---safework/documents/ policy/wcms_212074.pdf

13. León N. Diagnóstico situacional en seguridad y salud en el trabajo en Ecuador [en línea]. Lima: Instituto de Salud y Trabajo Perú; 2011 [consultado 19 feb 2016]. Disponible en: http://www.comunidadandina.org/camtandinos/OLA/ Documentos/Pdf/Diagnostico-SST-Ecuador_ISAT_2011.pdf

14. Gómez A, Suasnavas P. Incidencia de accidentes de trabajo declarados en Ecuador en el periodo 2011-2012. Cienc Trab. 2014; 17(52):49-53 [consultado 23 feb 2016]. Disponible en: http://www.cienciaytrabajo.cl/cyt/Paginas/ Incidencia-de-Accidentes-de-Trabajo-Declarados-en-Ecuador-en-elPeriodo-2011-2012.aspx

15. Gómez A, Suasnavas P, Freire L, Trujillo D. Accidentes de trabajo y enfermedades profesionales en Ecuador 2006-2014. Cuenca: Editorial Don Bosco; 2016.

16. Instituto Ecuatoriano de Seguridad Social. Estadísticas del Seguro de Riesgos del Trabajo-Seguro General de Riesgos de Trabajo [en línea]. Quito: Instituto Ecuatoriano de Seguridad Social; 2016 [consultado 20 feb 2016]. Disponible en: http://sart.iess.gob.ec/SRGP/indicadores_ecuador.php\#

17. Ecuador. Instituto Ecuatoriano de Seguridad Social. Ley de Seguridad Social: Ley 55. Registro Oficial, Suplemento 465 (30 nov 2001). Quito: Instituto Ecuatoriano de Seguridad Social; 2014.

18. Ecuador. Código del Trabajo. Registro Oficial, Suplemento 167 (26 sept 2012). Quito: Ministerio del Trabajo: 2012.

19. Organización Internacional de Trabajo. Convenio 121. Sobre las prestaciones en caso de accidentes del trabajo y enfermedades profesionales. En: 48 reunión CIT, 8 jul 1964; Ginebra, Suiza. Ginebra: OIT; 1964.

20. Organización de Estados Americanos (OEA)-Sistema de Información sobre Comercio Exterior (SICE) Comunidad Andina de Naciones. Decisión 584. Sustitución de la Decisión 547, Instrumento Andino de Seguridad y Salud en el Trabajo [en línea]. Washington: OEA; 2016 [consultado feb. 2016]. Disponible en: http://www.sice.oas.org/trade/junac/decisiones/DEC584s.asp

21. Comunidad Andina de Naciones. Resolución 957. Reglamento del Instrumento Andino de Seguridad y Salud en el Trabajo [en línea]. Quito: Instituto Ecuatoriano de Seguridad Social; 2005 [consultado feb 2016]. Disponible en: http://www.utm.edu.ec/unidadriesgos/documentos/resolucion957.pdf

22. Ecuador. Instituto Ecuatoriano de Seguridad Social. Reglamento del Seguro General de Riesgos del Trabajo. Resolución No. C.D. 513. Quito: Instituto Ecuatoriano de Seguridad Social; 2016.

23. Ecuador. Ministerio del Trabajo. Decreto Ejecutivo 2393 Reglamento de Seguridad y Salud de los Trabajadores y Mejoramiento del Medio Ambiente de Trabajo. Quito: Ministerio del Trabajo; 1986.

24. Ecuador. Instituto Ecuatoriano de Seguridad Social. Resolución No. C.D. 298 Reglamento General de Responsabilidad Patronal. Quito: Instituto Ecuatoriano de Seguridad Social; 2009.

25. Organización Iberoamericana de Seguridad Social. II Estrategia Iberoamericana de Seguridad y Salud en el Trabajo 2015-2020 (EISST). Madrid: OISS; 2014.

26. Eurostat. European Statistics on Accidents at Work (ESAW); Summary methodology [on line]. Luxembourg: Publications Office of European Union; 2013 [consultado 20 mar 2016]. Disponible en: http://ec.europa.eu/eurostat/ documents/3859598/5926181/KS-RA-12-102-EN.PDF/56cd35ba-1e8a-4af39f9a-b3c47611ff1c

27. Organización Internacional del Trabajo. XVI Conferencia Internacional de Estadisticos del Trabajo, 6-15 oct 1998; Ginebra, Suiza [en línea]. Ginebra: OIT; 1999 [consultado mar 2016]. Disponible en: http://www.lo.org/global/statistics-and-databases/meetings-and-events/international-conference-of-labourstatisticians/WCMS_087576/lang--es/index.htm

28. Ecuador. Instituto Ecuatoriano de Seguridad Social. Ley 55 de Seguridad Social. Quito: IESS; 2001.

29. nstituto Ecuatoriano de Seguridad Social. Rendición de cuentas para el periodo 2013-2015. Direcciones Provinciales de Riesgos del Trabajo [en linea]. Quito: IESS; 2015 [consultado 4 abr 2016]. Disponible en: https://www.iess.gob.ec/es/ rendicion-de-cuentas-2015.

30. Ecuador. Ley Orgánica de Transparencia y Acceso a la Información Pública. Ley 24. Registro Oficial Suplemento 337 (18 may 2004). Quito: Congreso Nacional; 2004.

31. Ecuador. Instituto Ecuatoriano de Seguridad Social. Resolución C.D. No. 333; Reglamento para el Sistema de Auditoria de Riesgos del Trabajo - SART. Quito: Instituto Ecuatoriano de Seguridad Social; 2010.

32. Ecuador. Instituto Ecuatoriano de Seguridad Social. Resolución C.D. No. 390; Reglamento del Seguro General de Riesgos del Trabajo. Quito: Instituto Ecuatoriano de Seguridad Social; 2011.

33. Salinas Tovar JS, López Rojas $P$, Soto Navarro MO, Caudillo Araujo DE, Sánchez Román FR y Borja-Aburto VH. El subregistro potencial de accidentes de trabajo en el Instituto Mexicano del Seguro Social. Salud Pública de México. 2004; 46: 204-209. 